\title{
Actividad sexual temprana y embarazo en la adolescencia: estado del arte
}

\author{
Luis Alfonso Mendoza Tascón ${ }^{1}$, Diana Isabel Claros Benítez ${ }^{2}$, Claudia Bibiana \\ Peñaranda Ospina. ${ }^{3}$ \\ ${ }^{1}$ Fundación Hospital San José de Buga. Buga, Colombia. Facultad Ciencias de la Salud, Programa de Medicina, Unidad \\ Central del Valle del Cauca. Tuluá, Colombia. ${ }^{2}$ Facultad Ciencias de la Salud, Programa de Medicina, Unidad Central del \\ Valle del Cauca. Tuluá, Colombia. ${ }^{3}$ Fundación Hospital San José de Buga. Buga, Colombia.
}

\section{RESUMEN}

La actividad sexual temprana en la adolescencia, representa un problema de salud pública por las consecuencias que conlleva, como el embarazo adolescente, el aumento de las infecciones de transmisión sexual, la infección por HIV/SIDA (segunda causa de muerte en los adolescentes) y los problemas familiares, económicos y sociales que se generan. El adolescente se ha convertido en una población de mayor riesgo en salud por la inequidad y las diferentes barreras a las que se enfrenta en materia de accesibilidad a los servicios de salud, en especial a los de salud sexual y reproductiva. Toda esta problemática ha hecho que esta población se vea enormemente afectada por morbilidades como las infecciones de transmisión sexual e infección por HIV/SIDA, a su vez con un mayor riesgo de morbilidad y mortalidad materna y perinatal. Es por ello que la educación sexual en el hogar, escuelas, colegios y servicios de salud, unido a la estimulación de habilidades para la vida, el retraso del inicio de la actividad sexual entre adolescente, la promoción de la abstinencia sexual y las prevención del embarazo y las infecciones de transmisión sexual, son una las estrategias más eficaces para enfrentar estas problemáticas.

\section{PALABRAS CLAVE: Adolescencia, actividad sexual, embarazo en adolescentes, prevención del embarazo}

\section{SUMMARY}

Early sexual activity in adolescence represent a public health problem for the consequences that entails, such as teenage pregnancy, the increase in sexually transmitted infections, HIV infection/AIDS (second cause of death among teenagers) and family problems, economic and social generated. The teenager has become a high-risk population in health inequality and the different barriers they face in terms of accessibility to health services, particularly sexual and reproductive health. All these problems has made this population being greatly affected by morbidities such as sexually transmitted infections and HIV/AIDS in turn to an increased risk of maternal and perinatal morbidity and mortality. That is why the sexual home education, schools, colleges and health services, together with the stimulation of life skills, delaying the onset of sexual activity among adolescent, promoting sexual abstinence and prevention of pregnancy and sexually transmitted infections are the most effective strategies to address these issues.

KEY WORDS: Adolescence, sexual activity, teenage pregnancy, preventing pregnancy 


\section{INTRODUCCIÓN}

Los Objetivos para el Desarrollo del Milenio (ODM) incorporan la sexualidad y la salud sexual y reproductiva (SSR), reconocida por la Organización Mundial de la Salud (OMS) como factor determinante para el bienestar y desarrollo de las personas, parejas, familias, comunidades y naciones, incluyendo la salud materna, reducción de la mortalidad infantil y lucha contra el VIH/SIDA (1).

La adolescencia es una etapa compleja, donde las decisiones y conductas que tomen los y las adolescentes en su SSR, dependerán de las oportunidades económicas, laborales, educativas, oferta de anticoncepción, situación socioeconómica, características personales, normas sociales e influencia del medio o contexto social en que viven y crecen $(2,3)$.

Las repercusiones que tiene el inicio temprano de la actividad sexual (AS), ha generado gran interés por parte de varias disciplinas (4), centrándose en identificar los diferentes factores asociados a este fenómeno $(5,6)$. Por otro lado, el embarazo adolescente se ha convertido en un importante problema de salud $(7,8)$. Es considerado una problemática, ya que los adolescentes tienen menos probabilidades de obtener un permiso legal y seguro para abortar en países donde el aborto está autorizado legalmente, con riesgo de obtener atención prenatal, parto y postnatal no calificada y problemas sociales, cuyo efecto se traduce en falta de oportunidades y aplanamiento de las perspectivas futuras de vida de la adolescente embarazada, su hijo y su familia (9).

\section{DEFINICIONES}

La adolescencia según la OMS es el período de la vida comprendido entre los 10 y 19 años (10-15). Se divide en tres etapas, con características diferentes, con formas distintas de enfrentar la sexualidad y un embarazo: 1. Adolescencia temprana (1014 años), 2. Adolescencia media (15-16 años) y 3. Adolescencia tardía (17-19 años) (10-16).

La sexualidad, es un aspecto central del ser humano, presente a lo largo de toda su vida. Incluye el sexo, identidades, papeles de género, erotismo, placer, intimidad, reproducción y orientación sexual. Se vivencia y se expresa a través de pensamientos, fantasías, deseos, creencias, actitudes, valores, conductas, prácticas y relaciones interpersonales. Puede incluir todas estas dimensiones, aunque no todas ellas se vivencian o se expresan siempre. Es esencial, constitutiva e inherente a todos los humanos, independiente de su condición física, mental y se expresa desde antes de nacer hasta la muerte, estando influenciada por la interacción de factores biológicos, psicológicos, sociales, económicos, políticos, culturales, éticos, legales, históricos, religiosos y espirituales (17).

Las relaciones sexuales, desde el punto de vista de la sexología, se pueden definir como el contacto físico entre personas fundamentalmente con el objeto de dar y/o recibir placer sexual, o con fines reproductivos. La relación sexual abarca muchas posibles actividades o formas de relación entre dos personas y no se limita sólo a coito o penetración. Incluye, en general, cualquier contacto que suponga excitación sexual con o sin orgasmo, como abrazos, masajes, caricias o besos en el cuerpo pero no en los genitales, caricias en la zona genital, sexo oral y penetración (con o sin eyaculación dentro de la vagina) (18).

\section{MAGNITUD DEL PROBLEMA}

La AS ocurre cada vez a más temprana edad, registrando promedios de inicio de 13,5-16 años, con edades tan tempranas cómo los 11 años $(7,8,19)$. En América Latina y El Caribe (ALC), aproximadamente $50 \%$ de los adolescentes menores de 17 años son sexualmente activos y 53-71\% de mujeres tuvieron relaciones sexuales antes de los 20 años (20). La edad del primer coito es de aproximadamente 15-16 años para las jóvenes de muchos países de ALC, mientras para otros, es tan temprana como los 10-12 años (21). Para Colombia en 2010, entre jóvenes de 20 y 24 años, el 14\% tuvo su primera relación antes de cumplir 15 años, $60 \%$ antes de cumplir 18 años y $82 \%$ antes de cumplir 20 años (22). Para Mendoza y cols (19), 15\% de los adolescentes y jóvenes han iniciado su AS en la adolescencia temprana, $44,8 \%$ en la adolescencia media y $40,2 \%$ en la adolescencia tardía. Holguín y cols (23), informan que $52,8 \%$ de adolescentes escolarizados han iniciado la AS a los 15 años (RI: 14-16 años), más tempranamente en varones (mediana 14 años; RI: 13-15 años) que en mujeres (mediana 15 años; RI: 14-16 años). El 23,8\% reportan tener más de una pareja sexual al año (varones 34\%; mujeres 12,7\%), con mayor número entre los varones (varones: mediana de 3; RI: 2-3; mínimo 0 y máximo 10; mujeres: mediana de 2; RI: 0-2; mínimo 0 y máximo 5). Las parejas sexuales en la primera relación sexual son de mayor edad en la mujeres que en los varones (varones: mediana 15 años; RI: 14-16 años; mínimo 10 y máximo 28 años; mujeres: mediana 18 años; RI: 16-20 años; mínimo 12 y máximo 32 años). Aproximadamente, una de cada cuatro mujeres adolescentes $(27,4 \%)$ tienen su primera relación con una persona de 20 a 32 años, mientras 3,4\% de varones con una persona de 20 a 28 años.

El embarazo en la adolescencia es un problema de origen multifactorial, con alto riesgo de complicaciones para la madre, el feto y el neonato, 
repercutiendo en los resultados perinatales (9). Los hijos de madres adolescentes representan aproximadamente el $11 \%$ de todos los nacimientos en el mundo, un $95 \%$ de ellas en países en desarrollo. La Organización Panamericana de la Salud (OPS), estima que $20 \%$ de nacimientos en la región son de madres menores de 20 años y $40 \%$ de estos embarazos no son deseados (24). El número de nacimientos de mujeres adolescentes de 15-19 años ha disminuido en todas las regiones del mundo entre 1990, 2000 y 2011. En el África Sub-Sahara son muy elevadas pasando de $1234 / 1.000$ mujeres (1990), 121/1.000 mujeres (2000), a 117/1.000 (2011) mujeres de 15-19 años. Para ALC el número de nacimientos en mujeres de 15-19 años por cada mil mujeres para los años 1990, 2000 y 2011, fue 86,86 y 76, mostrando un descenso para este último año (25). Cerca de 16 millones de adolescentes entre 15 y 19 años tienen un hijo cada año.

El $62,8 \%$ de los adolescentes en escuelas norteamericanas son sexualmente activos y cerca de 850.000 adolescentes resultan embarazadas cada año. En Estados Unidos de Norteamérica (EEUU) en 2004 , se estimó que $41,2 \%$ de embarazos ocurren en adolescentes de 15-19 años y $20 \%$ de estos, terminan en abortos inducidos. Sin embargo, en los últimos años han logrado reducir las tasas de embarazos en un 33\%, asociado a la reducción de tasas de AS y aumento del empleo más regular y prolongado de métodos anticonceptivos (26).

En Ecuador, 2 de cada 3 adolescentes de 15-19 años sin educación, son madres o están embarazadas por primera vez. En la última década la tendencia del incremento del embarazo en adolescentes menores de 15 años es de $74 \%$ y en mayores de 15 años de $9 \%$, siendo la tendencia de la fecundidad adolescente en Ecuador la más alta de la Región Andina, llegando a 100 por 1000 nacidos vivos (nv) $(27,28)$.

En Bolivia, cerca de $18 \%$ de adolescentes de 15-19 años ya son madres o están embarazadas; al menos la mitad de estos embarazos no fueron planificados al momento de la concepción (27).

Para Chile en 2009, el número total de nacidos vivos fue de 252.240 y de estos, el $16,14 \%$ correspondió a nacidos vivos de madres adolescentes; al desagregar por grupo etario, 15,7\% corresponden a adolescentes de $15-19$ años y $0,4 \%$ restante a adolescentes de 10-14 años (27).

Colombia registra una de las mayores tasas de embarazo adolescente de América Latina, donde 1 de cada 5 adolescentes entre 15 y 19 años ha estado embarazada $(27,28)$. Esta situación es más grave en víctimas del desplazamiento, donde $63 \%$ de las cuales han estado o están embarazadas de su primer hijo antes de cumplir los 19 años (28).
Holguin y cols (23), informan que para una ciudad colombiana, 7,2\% de las adolescentes entre 14-19 años escolarizadas encuestadas afirmó alguna vez haber estado embarazada.

\section{DETERMINANTES DE RIESGO PARA EL INICIO TEMPRANO DE LA ACTIVIDAD SEXUAL ENTRE ADOLESCENTES}

Son varios los determinantes que se han asociado con el inicio de la AS temprana, incluyendo los individuales, familiares, socioculturales y políticos. Estos se resumen en la Tabla I. Entre los determinantes individuales, se describen el sexo masculino, consumo de alcohol, tabaco y drogas cómo fuertemente vinculados con la AS de inicio temprano $(22,23,29,30-39)$. Entre los determinantes familiares, están la ausencia de uno o ambos padres, convivir con familias reconstituidas, pobre educación sexual en el hogar, mayor escolaridad de los padres (bachillerato, carrera técnica o profesional) y el hecho de que el padre o la madre trabajen, la nula o pobre relación con los padres, la mala comunicación en temas de sexualidad (23,40-46), entre otros factores como, un menor control y baja supervisión por parte de los padres, sin el establecimiento de límites y reglas a cerca de las actividades de noviazgo (47). Sin embargo, otros autores señalan que las conductas disciplinarias extremistas (déficit o exceso de normas) incrementan la probabilidad de inicio de AS entre adolescentes $(44,48)$. Entre los determinantes socio-culturales y políticos, el pertenecer a estratos socioeconómicos bajos (1 y 2) están asociados con AS temprana $(23,49)$. Sin embargo, varios estudios multivariados longitudinales coinciden en resaltar que el estado socioeconómico tiene muy poco efecto independiente sobre el momento del inicio de la AS (50), lo que sugiere que las intervenciones sobre las influencias del inicio de la AS temprana son eficaces.

La aprobación de las relaciones sexuales por parte de adolescentes se ve influenciada por su ambiente y contexto social, comportamiento pares o amigos y percepción que tienen los adolescentes de la presión existente por parte de sus pares y de sus amigos para tener o no relaciones sexuales a su edad $(23,51,52)$. No obstante, el apoyo mutuo para no tener sexo, es un factor protector para el inicio temprano de la AS (53). Brendgen y cols (54), hallaron que el rechazo de los pares se asocia indirectamente con un riesgo más elevado de AS temprana por su vinculación con la baja autoestima, pero sólo para las niñas. Mientras tanto, el abuso verbal por los profesores durante la infancia se asoció directamente con un mayor riesgo de tener AS temprana. 


\section{Tabla I \\ DETERMINANTES DE RIESGO PARA EL INICIO DE LA ACTIVIDAD SEXUAL TEMPRANA EN LA ADOLESCENCIA}

Determinantes individuales:

1. Sexo masculino.

2. Actitud permisiva hacia la AS en la adolescencia.

3. Consumo de licor.

4. Consumo de tabaco.

5. Consumo de drogas psicoactivas.

Determinantes familiares:

1. Falta de educación sexual por parte de la madre o la pobre comunicación en temas de sexualidad con la madre.

2. Antecedente de algún hermano o hermano haya sido padre o madre o haya estado en embarazo en la adolescencia.

3. Permisibilidad de los padres hacia la AS en la adolescencia.

4. Convivir con solo padre o con familias reconstituidas.

5. Un menor control y una baja supervisión por parte de los padres, sin el establecimiento de límites y reglas a cerca de las actividades de noviazgo.

6. Conductas disciplinarias extremistas (déficit o exceso de normas).

7. Relación nula o pobre entre el adolescente y sus padres.

8. Mayor escolaridad de los padres (bachillerato, carrera técnica o profesional) y el hecho de que el padre o la madre trabajen.

Determinantes socioculturales y políticos:

1. Pertenecer a estratos socioeconómicos bajos (estratos 1 y 2 ).

2. Comportamiento y presión por parte de compañeros, pares o amigos.

3. Influencia del ambiente y contexto social.

4. Pobre o nula educación en planificación familiar en colegios o servicios de salud.

5. Falta de políticas públicas, inequidades en el acceso a servicios de SSR y no consideración de los derechos sexuales y reproductivos de los adolescentes.

6. Erotización de la televisión de los medios de comunicación.

AS: Actividad sexual. SSR: Salud sexual y reproductiva.

\section{DETERMINANTES DE RIESGO PARA EL EM- BRAZO EN ADOLESCENTE}

Varios determinantes han sido implicados con el embarazo en adolescentes, y si bien muchos adolescentes tal vez deseen quedar embarazadas, muchos se producen en el contexto de violaciones de derechos humanos como el matrimonio infantil, relaciones sexuales forzadas o el abuso sexual (55). Este fenómeno se ha vinculado a múltiples determinantes individuales, familiares, socioculturales y políticos (Tabla II) (56). Hasta un $49 \%$ de adolescentes en embarazo se encuentran por fuera de escuelas y colegios, otras abandonan los estudios secundarios y $7,5 \%$ lo hacen por estar en embarazo y $5 \%$ por que se casan, entre otras razones. Las mujeres sin escolaridad tienen la edad de la primera unión marital a los 17,7 años (22). Un 81\% de adolescentes ha usado un método anticonceptivo alguna vez en su vida, pero sólo $10 \%$ los usa en su primera relación sexual (22), siendo este uno de los determinantes más importantes para embarazos precoces.

Entre los determinantes familiares, se describen la escolaridad de la madre de la adolescente inferior a 11 años de estudio, no educación sexual al adolescente por parte de la madre y no suministro de métodos de anticoncepción por parte de la madre del adolescente (56). La educación sexual adecuada impartida en hogares, colegios y servicios de salud, aunada a programas de "opciones de proyectos de vida", resolución de problemas y habilidades para la toma de decisiones, comunicación asertiva con los padres y equipos de salud en temas de sexualidad, han descritos como factores que reducen las tasas de embrazo en adolescentes (57). 


\section{Tabla II}

\section{DETERMINANTES DE RIESGO PARA EL EMBARAZO EN LA ADOLESCENCIA}

\section{Determinantes individuales:}

1. Realización por el adolescente de otra actividad diferente a estudiar.

2. Inicio de la AS muy temprana (15 años o menos).

3. No uso de métodos de planificación familiar en todas las relaciones sexuales.

4. Baja escolaridad.

5. Consumo alcohol.

6. Consumo de tabaco.

7. Consumo de drogas psicoactivas.

8. Falta de proyectos de vida.

Determinantes familiares:

1. Escolaridad materna inferior a 11 años de estudio.

2. No educación sexual al adolescente por parte de la madre.

3. No suministro de métodos de anticoncepción por parte de la madre del adolescente.

Determinantes socioculturales y políticos:

1. Estratos socioeconómicos bajos (estratos 1).

2. La ruralidad, origen étnico y oportunidades económicas limitadas para las adolescentes.

3. Insuficiente educación sexual por personal diferente a los padres (colegios y sector salud).

4. Pertenecer a familia no nuclear.

5. Madres que tuvieron su primer embarazo en la adolescencia.

6. Matrimonio infantil.

7. Abuso sexual.

8. Inequidades en el acceso a servicios de salud sexual y reproductiva.

9. Falta de políticas públicas de salud sexual y reproductiva para adolescentes.

10. No consideración de los derechos sexuales y reproductivos de las adolescentes.

11. Erotización de la televisión y los medios de comunicación.

AS: Actividad sexual.

Los determinantes socioculturales y políticos, incluyen diferentes problemáticas, como la socioeconómica, falta de educación, etnicidad, conformación familiar, primer embarazo de la madre en la adolescencia, entre otras (56,58-61). La información proporcionada a los adolescentes sobre anticoncepción, no da como resultado un aumento de las tasas de AS, ni un inicio más precoz en la edad de la AS, ni un mayor número de parejas sexuales (62). De hecho, si los adolescentes perciben obstáculos para la obtención de anticoncepción y condones, tienen mayor probabilidad de experimentar resultados negativos relacionados con la SSR (63). Dos trabajo en colegios, demostraron un retraso en el inicio de la AS empleando un enfoque de educación sexual más amplio, que incluía educación en anticoncepción $(64,65)$. Uno de los aspectos más relevantes, es la falta de servicios de salud para adolescentes, lo cual afecta de manera directa la educación en anticoncepción y accesibilidad a métodos de planificación familiar $(58,59)$.

\section{COMPLICACIONES Y CONSECUENCIAS DE LA ACTIVIDAD SEXUAL TEMPRANAY EL EMBARA- ZO EN LA ADOLESCENCIA}

El embarazo no deseado representa un riesgo entre adolescentes. El 59\% de embarazos terminan en nacimientos y $41 \%$ en abortos, $14 \%$ de ellos abortos involuntarios (66).

El $15 \%$ de abortos en el mundo (cifra que está alrededor de los 2 millones) ocurren en mujeres de 15-19 años de edad (25). El embarazo en la adolescencia, principalmente, en menores de 15 años, se puede asociar a complicaciones durante la gestación, parto y puerperio. Los aspectos biológicos (inmadurez ginecológica y anatómica de la pelvis, entre otros), psicológicos y socioculturales 
en la adolescente embarazada, repercuten en su resultado perinatal, con sus correspondientes consecuencias para la salud pública y de costos, tanto para la madre como para la familia y la sociedad (67). Los riesgos para resultados adversos entre las adolescentes embarazadas son mayores en comparación con las mujeres de más edad, incluyendo desnutrición, tabaquismo, abuso de sustancias, anemia, malaria, VIH/SIDA e ITS (68).

Las adolescentes se encuentran más desfavorecidas en el mantenimiento de un embarazo saludable, debido a un nivel de educación para la salud más pobre, falta de acceso a la atención prenatal, del parto, especializada u otro servicio de salud. A ello se suman los problemas económicos del embarazo y el parto. Un embarazo en una adolescente, puede contribuir a la dificultad para terminar la educación, aislamiento social, falta de apoyo familiar, oportunidades de empleo más pobres, perpetuación, feminización de la pobreza y transmisión intergeneracional de la pobreza consecuente. Esta confluencia de factores de riesgos intrínsecos y extrínsecos que las adolescentes embarazadas experimentan, pueden aumentar el riesgo de mortalidad y morbilidad grave durante el embarazo, el parto y el puerperio (25).

En cuanto a las morbilidades maternas entre las adolescentes se describen aumento de probabilidad de riesgo de hemorragia post-parto, obesidad, enfermedades mentales, preeclampsia-eclampsia, endometritis puerperal, infecciones sistémicas y muerte prematura (25). La mortalidad materna ha sido la segunda causa de muerte entre las adolescentes (15-19 años) en todo el mundo desde el año 2000, siendo más elevadas por debajo de los 20 años y después de los 30 años de edad. En mujeres de 15-19 años la tasa de mortalidad materna es de 260/100.000 mujeres y para las de 20-24 años es de 190/100.000 mujeres (25). Esto está asociado a la inequidad en educación, seguridad social en salud, además de otras barreras para que las gestantes adolescentes accedan al control prenatal (CPN). Un 30-40\% de adolescentes no se adhieren al CPN $(69,70)$, convirtiendo a la adolescencia es un determinante importante de la adherencia al CPN, el cual está vinculado con prematuridad, bajo peso al nacer y mortalidad neonatal (69). Por otro lado, los hijos de madres adolescentes tienen mayor riesgo de morbimortalidad, entre los cuales se destacan un mayor riesgo de prematuridad y bajo peso al nacer, sepsis, sífilis congénita y mortalidad entre los hijos de madres adolescentes (71-83).

\section{PREVENCIÓN DEL EMBARAZO EN LA ADO- LESCENCIA}

La necesidad insatisfecha de anticoncepción es alta entre adolescentes, asociado a una escasez de servicios de planificación familiar para este grupo. En África el $23,2 \%$ de mujeres en edad fecunda tienen una necesidad insatisfecha de anticonceptivos modernos. En Asia y ALC, las necesidades insatisfechas representan un $10,9 \%$ y un $10,4 \%$, respectivamente (84).

Dos intervenciones han sido eficaces en la reducción de embarazos entre adolescentes, la abstinencia sexual y el mejor uso de anticonceptivos, con los cuales se reduce un $38 \%$ del riesgo global de embarazos, mientras un $86 \%$ de esta caída se atribuye al uso de anticonceptivos. Entre los adolescentes de 15-17 años, 77\% de la reducción del riesgo de embarazos, es atribuida al uso de anticonceptivos y $23 \%$ a la abstinencia sexual (85). Esto ha hecho sospechar que la promoción de la abstinencia sexual, no es la principal estrategia para prevenir embarazos en adolescentes (86), dejando a los anticonceptivos como la mejor forma de prevención.

Para iniciar la anticoncepción del adolescente se debe preparar el escenario y tener en cuenta varios aspectos: el consentimiento y protección de la confidencialidad, asesoramiento, participación del adolescente, elección de los métodos para prevención de ITS y embarazos (66). Los médicos deben recordar a los adolescentes que, con todos los métodos anticonceptivos hormonales (incluyendo la inyección de progestina) que buscan prevenir embarazos, se deben utilizar condones para protegerse de las ITS. Las Tablas III y IV, resumen los métodos anticonceptivos que más frecuentemente son empleados por los adolescentes, recomendando siempre un método que tenga menor tasa de fracasos, mejor adherencia y siempre usando un método de barrera tipo condón.

\section{CONCLUSIONES}

Son diferentes los determinantes asociados al inicio temprano de la AS y embarazo entre adolescentes, entre ellos las inequidades, barreras de acceso a los servicios SSR y políticas en salud. Las consecuencias de estos dos fenómenos son graves para la salud pública, afectando las dimensiones familiar, social, de salud y económica. Si queremos impactar sobre la salud de los adolescentes, en especial sobre su SSR, se deben reducir estas inequidades, mejorar la accesibilidad de los adolescentes a los servicios de salud, estableciendo con ellos proyectos de vida a través del desarrollo de sus habilidades, brindar una adecuada educación sexual y mejorando el acceso a los métodos de prevención del embarazo y las ITS. 


\section{Tabla III}

ANTICONCEPCIÓN EN ADOLESCENTES

\begin{tabular}{|c|c|c|c|c|c|}
\hline Método & $\begin{array}{l}\text { Tasa de } \\
\text { fracaso }\end{array}$ & $\begin{array}{l}\text { Tiempo de } \\
\text { cambio o de } \\
\text { nueva dosis }\end{array}$ & $\begin{array}{l}\text { Periodo de } \\
\text { protección }\end{array}$ & $\begin{array}{l}\text { Efectos } \\
\text { colaterales }\end{array}$ & $\begin{array}{c}\text { Mejor } \\
\text { recomendado } \\
\text { en adolescentes }\end{array}$ \\
\hline $\begin{array}{l}\text { Implante de proges- } \\
\text { tágeno -etonoges- } \\
\text { trel- (Implanon } \AA \text { y } \\
\text { Nexplanon } \AA)\end{array}$ & $<1 \%$ & 3 años & Prolongado & $\begin{array}{l}\text { Labilidad emocional, } \\
\text { aumento de peso, } \\
\text { cefalea, acné, san- } \\
\text { grado irregular }\end{array}$ & $\mathrm{Si}$ \\
\hline
\end{tabular}

Anticoncepción intrauterina o dispositivos intrauterinos (de cobre: TCu380A* y MLCu375; y liberadores de levonorgestrel: por 52 mg, Mirena ${ }^{\star \star}$ y por 13,5 mg, Skyla®)

\begin{tabular}{|c|c|c|c|c|c|}
\hline $\begin{array}{l}\text { Inyecciones de pro- } \\
\text { gestina - acetato de } \\
\text { medroxiprogestero- } \\
\text { na - (Depo-Prove- } \\
\mathrm{ra}(\mathbb{R})\end{array}$ & $\begin{array}{l}\text { Uso correcto } \\
\text { y sostenible: } \\
0,2-1 \% \text {; uso } \\
\text { común 3-6\% }\end{array}$ & 13 semanas & Corta & $\begin{array}{l}\text { Ganancia de peso, } \\
\text { interferencia con el } \\
\text { incremento normal, } \\
\text { cefalea, mastalgia, } \\
\text { caída del cabello, } \\
\text { cambios de la libido }\end{array}$ & $\mathrm{Si}$ \\
\hline $\begin{array}{l}\text { Anticonceptivos } \\
\text { orales combinados } \\
\text { (Combinación de } \\
\text { estrógeno y proges- } \\
\text { tina) }\end{array}$ & $\begin{array}{l}\text { Uso correcto } \\
\text { y sostenible: } \\
0,3-1 \% \text {; uso } \\
\text { común } 8-9 \%\end{array}$ & 28 días & Corta & $\begin{array}{l}\text { Sangrado irregular, } \\
\text { cefalea, náuseas }\end{array}$ & $\mathrm{Si}$ \\
\hline
\end{tabular}

Anticonceptivo transdérmico -0,6 mg de norelgestromina y $0,75 \mathrm{mg}$ de etinilestradiol- (Ortho Evra®)

1\%
*TCu380A: $10 \quad$ Prolongada
años
**Mirena: 5 años

Expulsión del dispo-

$\mathrm{Si}$ sitivo, experiencias
de dolor y malestar

Mastalgia, cefalea,

Si náuseas, hemorragia uterina, desplazamiento del parche, hiperpigmentación de la piel, dermatitis de contacto, otras irritaciones de la piel

\begin{tabular}{|c|c|c|c|c|c|}
\hline $\begin{array}{l}\text { Píldoras de proges- } \\
\text { terona sola }\end{array}$ & $\begin{array}{l}\text { Uso correcto } \\
\text { y sostenible: } \\
1 \% \text {; uso } \\
\text { común } \\
3-12 \%\end{array}$ & $\begin{array}{l}\text { 4-22 horas } \\
\text { previas al coito }\end{array}$ & Corta & Náuseas y vómito & $\mathrm{Si}$ \\
\hline $\begin{array}{l}\text { Anticonceptivo de } \\
\text { emergencia (proges- } \\
\text { tina sóla con levo- } \\
\text { norgestrel } 1,5 \mathrm{mg} \text { o } \\
\text { combinada de estró- } \\
\text { geno y progestina) }\end{array}$ & $6-48 \%$ & $\begin{array}{l}\text { Iniciarse antes } \\
\text { de } 5 \text { días } \\
\text { después de la } \\
\text { relación sexual }\end{array}$ & Corta & $\begin{array}{l}\text { Náuseas, vómito, } \\
\text { cefalea, menstrua- } \\
\text { ción dolorosa, mas- } \\
\text { talgia }\end{array}$ & $\mathrm{Si}$ \\
\hline
\end{tabular}




\section{MÉTODOS DE BARRERA Y OTROS MÉTODOS DE ANTICONCEPCIÓN EN ADOLESCENTES}

\begin{tabular}{|c|c|c|c|c|c|}
\hline Método & $\begin{array}{l}\text { Tasa de } \\
\text { fracaso }\end{array}$ & $\begin{array}{l}\text { Tiempo de } \\
\text { cambio o de } \\
\text { nueva dosis }\end{array}$ & $\begin{array}{l}\text { Periodo de } \\
\text { protección }\end{array}$ & Efectos colaterales & $\begin{array}{l}\text { Mejor reco- } \\
\text { mendado en } \\
\text { adolescentes }\end{array}$ \\
\hline $\begin{array}{l}\text { Condones masculi- } \\
\text { nos }\end{array}$ & $\begin{array}{l}\text { Uso correcto } \\
\text { y sostenible: } \\
2 \% \text {; uso } \\
\text { común } 15- \\
18 \%\end{array}$ & $\begin{array}{l}\text { Inmediato con } \\
\text { cada relación } \\
\text { sexual }\end{array}$ & Corta & Alergia al látex & $\mathrm{Si}$ \\
\hline $\begin{array}{l}\text { Condones femeni- } \\
\text { nos }\end{array}$ & $\begin{array}{l}\text { Uso correcto } \\
\text { y sostenible: } \\
5-10 \% \text {; uso } \\
\text { común } 21 \%\end{array}$ & $\begin{array}{l}\text { Inmediato con } \\
\text { cada relación } \\
\text { sexual }\end{array}$ & Corta & Alergia al látex & $\mathrm{Si}$ \\
\hline 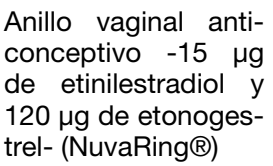 & $9 \%$ & 28-35 días & Corta & $\begin{array}{l}\text { Mastalgia, cefalea, } \\
\text { náuseas, hemorra- } \\
\text { gia por disrrupción } \\
\text { o manchado, expul- } \\
\text { sión del dispositivo }\end{array}$ & No \\
\hline $\begin{array}{l}\text { Espermicidas vagi- } \\
\text { nales (Nonoxinol-9) }\end{array}$ & $\begin{array}{l}\text { Uso correcto } \\
\text { y sostenible: } \\
15 \% \text {; uso } \\
\text { común } 25 \%\end{array}$ & $\begin{array}{l}\text { Inmediato con } \\
\text { cada relación } \\
\text { sexual }\end{array}$ & Corta & $\begin{array}{l}\text { No suelen reco- } \\
\text { mendarse como un } \\
\text { anticonceptivo de } \\
\text { primera elección en } \\
\text { adolescentes sanas. }\end{array}$ & No \\
\hline $\begin{array}{l}\text { Diafragma, capu- } \\
\text { chón cervical y es- } \\
\text { ponja anticonceptiva }\end{array}$ & $\begin{array}{l}\text { Uso correcto } \\
\text { y sostenible: } \\
\text { 9-12\%; uso } \\
\text { común 20- } \\
25 \%\end{array}$ & $\begin{array}{l}\text { Inmediato con } \\
\text { cada relación } \\
\text { sexual }\end{array}$ & Corta & $\begin{array}{l}\text { No suelen reco- } \\
\text { mendarse como un } \\
\text { anticonceptivo de } \\
\text { primera elección en } \\
\text { adolescentes sanas }\end{array}$ & No \\
\hline $\begin{array}{l}\text { Conocimiento de la } \\
\text { fertilidad y otros mé- } \\
\text { todos de abstinencia } \\
\text { periódica }\end{array}$ & $\begin{array}{l}\text { Uso correcto } \\
\text { y sostenible: } \\
5 \% \text {; uso } \\
\text { común } 12 \%\end{array}$ & No aplica & No aplica & No tiene & No \\
\hline
\end{tabular}

\section{REFERENCIAS}

1. Organización Panamericana de la Salud. Salud Sexual para el Milenio. Declaración y Documento Técnico. Washington, DC: OPS, 2009. Acceso: 10/12/2015. Disponible en: http://www1.paho.org/hq/ dmdocuments/2010/Salud\%20Sexual\%20para\%20 el\%20Milenio.pdf.

2. Bearinger L, Sieving R, Ferguson J, Sharma V. Global perspective on the sexual and reproductive health of adolescents: patterns, prevention, and potential. Lancet 2007;369:1220-31.

3. Trave T, Maya M, Vilaplana E. Repercusión neonatal del embarazo en adolescentes An Esp Pediatr 1989;3L(4):368-71.

4. Gillmore MR, Archibald ME, Morrison DM, Wilsdon A, Wells EA, Hoppe M, et al. Teen Sexual Behavior:
Applicability of the Theory of Reasoned Action. J Marriage Family 2002;64(4):885-98.

5. Carvajal SC, Parcel GS, Banspach SW, Basen Engquist K, Coyle KK, Kirby D, et al. Psychosocial predictors of delay of first sexual intercourse by adolescents. Health Psychology 1999;18:443-52.

6. Pedersen W, Samuelsen SO, Wichstrøm L. Intercourse debut age: Poor resources, problem behavior or romantic appeal? J Sex Res 2003;40(4):333-45.

7. Lee MC, Suhng LA, Lu TH, Chou MC. Association of parental characteristics with adverse outcomes of adolescent pregnancy. Fam Pract 1998;15:336-42.

8. Klima CS. Centering pregnancy: a model for pregnant adolescents. J Midwifery Womens Health 2003;48:220-5.

9. WHO guidelines on preventing early pregnancy and poor reproductive outcomes among adolescents in 
developing countries. World Health Organization 2011. Acceso: 10/12/15. Disponible en: http://www. who.int/maternal__child_adolescent/documents/preventing_early_pregnancy/en/index.html.

10. Issler JR. Embarazo en la adolescencia. Revista de posgrado de la VI cátedra de Medicina 2001;107:1123.

11. Serra FG, Spaciuk SM, Morales E, Arce GM, Avalos ME, Gómez VY. Riesgo reproductivo del adolescente en Corrientes. Revista de posgrado de la VI cátedra de Medicina 2002;115:24-5.

12. Balestena JM, Balestena SG. Impacto de la menarquía en los resultados maternos perinatales en la adolescencia. RCOG [en línea] 2005 [2 de enero de 2012]; 31(1).URL disponible en:http://www.bvs.sld.cu/ revistas/gin/vol31_1_05/gin02105. htm

13. Laffita A, Ariosa JM, Cutié JR. Adolescencia e interrupciones del embarazo. RCOG [en línea] 2004 [2 de enero de 2012]; 30(1).URL disponible en: http://www. bvs.sld.cu/revistas/gin/vol30_1_04/gin04104.htm

14. Valdes S, Essien J, Bardales J, Saavedra D, Bardales $\mathrm{E}$. Embarazo en la adolescencia. Incidencia, riesgos y complicaciones. RCOG. [en línea] 2002 [2 de enero de 2012 de noviembre de 2005]; 28(2). URL disponible en:http://www.bvs.sld.cu/revistas/gin/vol28_2_02/ gin04202.htm

15. Fernández LS, Carro E, Osesa D, Pérez J. Caracterización del recién nacido en una muestra de gestantes adolescentes. RCOG [en línea] 2004 [2 de enero de 2012]; 30(2). URL disponible en:http://www.bvs.sld. cu/revistas/gin/vol30_2_04/gin03204.htm.).

16. Romero MI, Maddaleno M, Silber TJ, Munist M. Salud reproductiva. En: Silber TJ, Munist MM, Maddaleno M, Suarez Ojeda. EN: Manual de medicina de la adolescencia. EEUU: Publicación de la OPS 1992:473518.

17. Organización Mundial de la Salud - OMS. (2006). Defining sexual health: report of a technical consultation on sexual health. Acceso: 10/12/2015. Disponible en: http://www.who.int/reproductivehealth/publications/ sexual_health/defining_sexual_healt h.pdf.

18. Sexualidad - Seguridad. Información práctica sobre las relaciones sexuales y la sexualidad. Acceso: 10/12/2015. Disponible en: http://sexualidadseguridad.blogspot.com.co/2010/03/definicion-de-relacionsexual.html.

19. Mendoza LA, Arias M, Pedroza M, Micolta P, Ramírez $\mathrm{A}$, Cáceres $\mathrm{Ch}$, et al. Actividad sexual en adolescencia temprana: problema de salud pública en una ciudad colombiana. Rev Chil Obstet Ginecol 2012;77(4):2719.

20. Schutt-Aine J, Maddaleno M. (2003). Salud sexual y desarrollo de adolescentes y jóvenes en las Américas: Implicaciones en programas y políticas. (sitio en Internet). OPS, Washington, DC. Acceso: 22-122015. Disponible: www.paho.org/Spanish/HPP/HPF/ ADOL/SSRA.pdf.

21. García R, Cortés A, Vila L, Hernández M, Mesquia A. Comportamiento sexual y uso del preservativo en adolescentes y jóvenes de un área de salud. Rev Cubana Med Gen Integr 2006;22(1). Acceso: 13-12-2015. Disponible en: bvs.sld.cu/revistas/mgi/ vol22_1_06/mgi03106.pdf.

22. Profamilia, Ministerio de la Protección Social República de Colombia, Bienestar Familiar y United States Agency International Development, USAID del Pueblo de los estados Unidos. Conocimientos del VIH y otras ETS, Encuesta Nacional de Demografía y salud ENDS 2010. Informe de prensa, 2010. Acceso: 18-12-2015. Disponible en: http://www.profamilia.org.co/encuestas/Profamilia/ Profamilia/index.php?option=com _ content\&view $=$ arti cle\&id=62\&ltemid $=9$.

23. Holguín YP, Mendoza LA, Esquivel CM, Sánchez R, Daraviña $A D$, Acuña $M$. Factores asociados al inicio de la actividad sexual en adolescentes de Tuluá, Colombia. Rev Chil Obstet Ginecol 2013;78(3):209-19.

24. Organización Panamericana de Salud, Salud en las Américas, 2007. Acceso: 10-01-2015. Disponible en: http://bvs.per.paho.org/videosdigitales/matedu/RegionAmericas_fgonzales.pdf)

25. United Nations. Millennium Development Goals Report 2014. New York: United Nations, 2014. . Acceso: 17/12/2015. Disponible en: http://www.un.org/ millenniumgoals/2014\%20MDG\%20report/MDG\%20 2014\%20English\%20web.pdf.

26. Contraception and Adolescents. Committee on Adolescence. THE AMERICAN ACADEMY OF PEDIATRICS. Pediatrics 2007; 120;1135-48.

27. Plan Andino para la prevención del embarazo en adolescentes. Acceso 12-11-2015. Disponible en: http:// www.planandinopea.org/?q=node/60.

28. United States Agency for International Development, Profamilia. Salud sexual y reproductiva en zonas marginadas: situación de las mujeres desplazadas 2005. Bogotá: United States Agency International Development, Profamilia; 2005. Acceso 12-11-2015. Disponible en: http://www.unicef.org/lac/INVERSION_EN_ ADOLESCENTES(3).pdf.

29. Carvajal SC, Parcel GS, Banspach SW, Basen Engquist K, Coyle KK, Kirby D, et al. Psychosocial predictors of delay of first sexual intercourse by adolescents. Health Psychology 1999;18:443-52.

30. Rosenthal D, Moore S, Flynn I. Adolescent self-efficacy, self-esteem and sexual risk-taking. J Community \& Applied Social Psychol 1991;1:77-88.

31. AAP Committee on Substance Abuse. Alcohol use and abuse: a pediatric concern. Pediatrics 1995;95:43942.

32. Kirby D. Emerging answers: Research findings on programs to reduce teen pregnancy. Washington, DC: National Campaign to Prevent Teen Pregnancy; 2001:13-72.

33. Kinsman S, Romer D, Furstenberg F, Schwarz D. Early sexual initiation: the role of peer norms. Pediatrics 1998;102:1185-92.

34. Hingson R, Heeren T, Winter M, Wechsler H. Early age of first drunkenness as a factor in college students' unplanned and unprotected sex attributable to drinking. Pediatrics 2003;111:34-41.

35. Dunn M, Bartee R, Perko M. Self-reported alcohol use and sexual behaviors of adolescents. Psychol Rep 2003;92:339-48.

36. Strasburger V. Alcohol advertising and adolescents. Pediatr Clin North Am 2002;49:353-76.

37. Strasburger V. Getting teenagers to say no to sex, drugs, and violence in the new millennium. Med Clin North Am 2000;84:787-810.

38. Brown R. Risk factors for substance abuse in adolescents. Pediatr Clin North Am 2002;49:247-55.

39. Ree D, Argys L, Averett S. New evidence on the relationship between substance use and adolescent sexual behavior. J Health Econ 2001;20:835-45. 
40. Andrade P, Betancourt D, Palacios JR. Factores familiares asociados a la conducta sexual en adolescentes. Rev Colomb Psicol 2006;15:91-101.

41. Brewester KL, Billy JOG, Grady WR. Social context and adolescent behavior: The impact of community on the transition to sexual activity. Social Forces 1993;71:713-40.

42. Capaldi DM, Crosby L, Stoolmiller M. Predicting the timing of first sexual intercourse for at risk adolescent males. Child Development 1996;67:344-59.

43. Forste RT, Heaton TB. Initiation of sexual activity among female adolescent. Youth Soc 1998;19:25068.

44. Caffery TE. A study on the effects of family functioning on attachment security in college students. Dissertation Abstracts International: section $\mathrm{B}$ : the sciences and engineering. 2000;60(10-B).

45. Fisher TD. Parent-child communication about sex and young adolescent's sexual knowledge and attitudes. Adolescence 1986;21:517-27.

46. Fox GL, Inazu JK. Patterns and outcomes of mother daughter communication about sexuality. J Soc Issues 1980;36:7-29.

47. Hovell M, Sipan C, Blumberg E, Atkins C, Hofstetter RC, Kreitner S. Family influences on Latino and Anglo adolescent's sexual behavior. J Marriage Fam 1994;53:973-86.

48. Upchurch DM, Aneshensel CS, Sucoff CA, LevyStorms L. Neighborhood and family contexts of adolescent sexual activity. J Marriage Fam 1999;61:920-33.

49. Kirby D. Antecedents of adolescent initiation of sex, contraceptive use, and pregnancy. Am J Health Behavior 2002;26(6):473-85

50. Zimmer-Gembeck MJ, Helfand M. Ten years of longitudinal research on U.S. adolescent sexual behavior: developmental correlates of sexual intercourse, and the importance of age, gender and ethnic background. Developmental Rev 2008;28(2):153-224.

51. Vargas E, Barrera F. ¿Es la autoestima una variable relevante para los programas de prevención del inicio temprano de la actividad sexual? Acta Colomb Psicol 2005;13:133-61.

52. Laflin MT, Wang J, Barry M. A longitudinal study of adolescent transition from virgin to nonvirgin status. J Adol Health 2008;42(3):228-36.

53. Robinson G. Families, generations and self: Conflict, loyalty and recognition in an Australian aboriginal society. Ethos 1997;25(3):303-32.

54. Brendgen M, Wanner B, Vitaro F. Peer and teacher effects on the early onset of sexual intercourse. Am J Public Health 2007;97:2070-5.

55. Chandra-Mouli V, Camacho A.V., Michaud P.A. WHO guidelines on preventing early pregnancy and poor reproductive outcomes among adolescents in developing countries. J Adolesc Health 2013;52:517-22.

56. Sánchez YA, Mendoza LA, Grisales MB, Ceballos LY, Bustamante JC, Muriel E, et al. Características poblacionales y factores asociados a embarazo en mujeres adolescentes de Tuluá, Colombia. Rev Chil Obstet Ginecol 2013; 78(4): $269-281$.

57. Tripp J, Viner R. ABC of adolescence sexual health, contraception, and teenage pregnancy. BMJ 2005;330:590-3.

58. World Health Organization. Position paper on mainstreaming adolescent pregnancy in efforts to make pregnancy safer [Internet]. Geneva: World Health Or- ganization, 2010. Acceso: 20-12-2015. Disponible en: http://www.who.int

59. Rani M, Lule E. Exploring the socioeconomic dimension of adolescent reproductive health: a multicountry analysis. Int Fam Plan Perspect 2004;30:110-17.

60. Alba LE. Salud de la adolescencia en Colombia: bases para una medicina de prevención. Univ Méd Bogotá (Colombia) 2010;51:29-42.

61. Santos MI, Rosario F. A score for assessing the risk of first-time adolescent pregnancy. Family Practice 2011;28:482-8.

62. Kirby DB, Laris BA, Rolleri LA. Sex and HIV education programs: their impact on sexual behaviors of young people throughout the world. J Adolesc Health 2007;40:206-17.

63. Guttmacher S, Lieberman L, Ward D, Freudenberg $\mathrm{N}$, Radosh A, Des Jarlais D. Condom availability in New York City public high schools: relationships to condom use and sexual behavior. Am J Public Health 1997;87:1427-33.

64. Kirby D, Barth RP, Leland N, Fetro JV. Reducing the risk: impact of a new curriculum on sexual risk-taking. Fam Plann Perspect 1991;23:253-63.

65. Zabin LS, Hirsch MB, Smith EA, Streett R, Hardy JB. Evaluation of a pregnancy prevention program for urban teenagers. Fam Plann Perspect 1986;18:119-26.

66. Ott M.A., Sucato G.S., and Committee on adolescence Pediatrics 2014. Contraception for Adolescents. Acceso: 21/12/2015. Disponible: http://pediatrics.aappublications.org/content/pediatrics/early/2014/09/24/ peds.2014-2299.full.pdf.

67. Parada A, Becerra D, Villacis C. Obstetricia Integral Siglo XXI: Embarazo en la Adolescencia. Universidad Nacional de Colombia 2009.

68. Vogel J., Pileggi C., Chandra V., Nogueira V., Paulo J., Chou D., et al. Millennium Development Goal 5 and adolescents: looking back, moving forward. Arch Dis Child 2015;100 (Suppl 1):s43-s47. Acceso 17/12/2015. Disponible en: http://adc.bmj.com/content/100/Suppl_1/S43.full.pdf+html.

69. Mendoza LA, Arias MD, Peñaranda CB, Mendoza LI, Manzano S, Varela AM. Influencia de la adolescencia y su entorno en la adherencia al control prenatal e impacto sobre la prematuridad, bajo peso al nacer y mortalidad neonatal. Rev Chil Obstet Ginecol 2015;80(4): 306-15.

70. Gallego LI, Vélez GA, Agudelo B. Panorama de la mortalidad materna. Colombia 2008;43(5 Pt 2):17816.

71. Mendoza LA, Arias M, Mendoza L.. Hijo de madre adolescente: riesgos, morbilidad y mortalidad neonatal. Rev Chil Obstet Ginecol 2012;77(5):375-82.

72. Davidson NW, Felice ME. Adolescent pregnancy. In: Friedman SB, Fisher M, Schonberg SK, eds. Comprehensive Adolescent Health Care. St Louis, MO: Quality Medical Publishing Inc; 1992:1026-40.

73. Chotigeat U, Sawasdiworn S. Comparison outcomes of sick babies born to teenage mothers with those born to adult mothers. J Med Assoc Thai 2011;94(Suppl.3):S27-S34.

74. Klerman LV. Adolescent pregnancy and parenting: controversies of the past and lessons for the future. $\mathrm{J}$ Adolesc Health 1993;14:553-61.

75. Goldenberg RL, Klerman LV. Adolescent pregnancy- another look. N Engl J Med 1995;332:1161-2. 
76. Fraser AM, Brockert JE, Ward RH. Association of young maternal age with adverse reproductive outcomes. N Engl J Med 1995;332:1113-7.

77. Blankson ML, Cliver SP, Goldenberg RL, Hickey CA, Jin J, Dubard MB. Health behavior and outcomes in sequential pregnancies of black and white adolescents. JAMA 1993;269:1401-3.

78. Islas LP, Cardiel L, Figueroa JF. Recién nacidos de madres adolescentes. Problema actual. Rev Mex Pediatr 2010;77:152-5.

79. McDevitt TM, Adlakha A, Fowler TB, et al. Trends in adolescent fertility and contraceptive use in the developing world. [IPC/95-1]. Washington, DC: U.S. Bureau of the Census, 1996.

80. Martin R, Buttari I, Macias H, et al. A.I.D.'s Family Planning Program in Honduras. [A.I.D. Technical Report; no. 9]. Washington, DC: Agency for International Development, 1993.

81. Doig J. Indicadores perinatales en hijos de madres adolescentes del Instituto Especializado Materno
Perinatal durante el año 2003. Rev Peruana Pediatr 2006;59:6-11.

82. Donoso E, Becker J, Villarroel L. Natalidad y riesgo reproductivo en adolescentes de Chile, 1990-1999. Rev Panam Salud Pública 2003;14:3-8.

83. United Nations. The Health Rationale for Family Planning: timing of births and child survival. Nueva York, NY: The UN, 1994.

84. Organización Mundial de la Salud. Planificación familiar. Nota descriptiva 351. Mayo 2015. Acceso: 06-012016. Disponible en: http://www.who.int/mediacentre/ factsheets/fs351/es/

85. Santelli JS, Lindberg LD, Finer LB, Singh S. Explaining recent declines in adolescent pregnancy in the United States: The contribution of abstinence and improved contraceptive use. Am J Public Health 2007;97:150-6.

86. Pinkerton SD. A relative risk-based, disease-specific definition of sexual abstinence failure rates. Health Educ Behav 2001;28:10-20. 